\title{
Seroprevalencia de leishmaniosis visceral canina en la comuna 8 de Neiva y en cuatro municipios de Huila, Colombia
}

\author{
José Fernández ${ }^{1}$, Felio Bello ${ }^{2}$, Myriam Consuelo López ${ }^{3}$, Ligia Inés Moncada ${ }^{3}$, Jimmy \\ Jolman Vargas ${ }^{4}$, Martha Stella Ayala ${ }^{5}$, Rubén Santiago Nicholls ${ }^{3,5}$, Carlos Alberto Lozano ${ }^{6}$ \\ ${ }^{1}$ Grupo de Epidemiología y Salud Pública, Facultad de Medicina Veterinaria, Universidad de La Salle, \\ Bogotá, D. C., Colombia. \\ ${ }^{2}$ Laboratorio de Entomología, Biología Celular y Genética, Departamento de Ciencias Básicas, Universidad \\ de La Salle, Bogotá, D. C., Colombia. \\ ${ }^{3}$ Departamento de Salud Pública, Facultad de Medicina, Universidad Nacional de Colombia, Bogotá, D. C., \\ Colombia. \\ ${ }^{4}$ Departamento de Ciencias para la Salud Animal, Facultad de Medicina Veterinaria y de Zootecnia, \\ Universidad Nacional de Colombia, Bogotá, D. C., Colombia. \\ ${ }^{5}$ Laboratorio de Parasitología, Instituto Nacional de Salud, Bogotá, D. C., Colombia. \\ ${ }^{6}$ Secretaría Departamental de Salud del Huila, Neiva, Colombia.
}

Introducción. La leishmaniosis visceral canina en áreas endémicas de Colombia se constituye en un factor de riesgo para la salud pública, dado el carácter zoonótico de la enfermedad. Durante el año 2004 se registraron 96 casos de leishmaniosis visceral humana en el país, de los cuales cinco correspondieron al departamento del Huila, zona de influencia del vector Lutzomyia longipalpis.

Objetivo. Determinar la prevalencia de anticuerpos IgG contra Leishmania chagasi en sueros de caninos recolectados en los municipios de Rivera, Palermo, Villavieja, Algeciras y la comuna 8 de la ciudad de Neiva, Huila, Colombia.

Materiales y métodos. Se realizó una encuesta epidemiológica a los propietarios de 610 caninos y previo consentimiento se llevó a cabo examen clínico general y recolección de muestra de sangre de cada una de las mascotas. Los sueros fueron analizados mediante el test de ELISA empleando como antígeno promastigotes de Leishmania chagasi, de la cepa MHOM/CO/84/CL044B. El análisis de datos empleó estadística descriptiva.

Resultados. En la población canina $85 \%$ fueron mestizos, la edad promedio fue 2,5 años y $67,3 \%$ de los animales fueron machos. Al examen clínico las manifestaciones más frecuentes fueron: onicogrifosis $24,3 \%$, linfadenitis $10 \%$ y lesiones cutáneas $5 \%$. La frecuencia de anticuerpos IgG contra Leishmania chagasi en la comuna 8 de la ciudad de Neiva fue 28,1\%, en Villavieja $28 \%$, en Rivera 14,9\%, en Palermo 10\% y en Algeciras 5,1\%. Adicionalmente, se observó un promedio de cinco personas convivientes por canino seropositivo.

Conclusiones. Estos datos demuestran la exposición de los caninos que habitan los ambientes rurales y urbanos de las zonas en estudio a Leishmania chagasi y deben tenerse en cuenta para el fortalecimiento de los programas de control y prevención de esta zoonosis en el Sistema General de Seguridad Social en Salud.

Palabras clave: enfermedades de los perros, Leishmania, test de ELISA, Colombia.

Seroprevalence of canine visceral leishmaniasis in sector 8 of Neiva and in four municipalities of Huila, Colombia

Introduction. Canine visceral leishmaniasis in endemic areas of Colombia could be a public health risk factor given the zoonotic nature of the disease. Ninety-six human cases of visceral leishmaniasis were reported in Colombia in 2004, 5 of them in Huila, where Lutzomyia longipalpis has been incriminated as the main vector species.

Objective. To determine the prevalence of IgG antibodies against Leishmania chagasi in dogs from the sector 8 of the city of Neiva and the from the towns of Villavieja, Algeciras, Palermo and Rivera located in Huila, Colombia. 


\begin{abstract}
Materials and methods. An epidemiological survey was carried out in 610 dogs, which included clinical examination and venopuncture for obtaining blood samples. Authorization was obtained from the dog owners. The sera were analyzed by the ELISA test with promastigotes of the $L$. chagasi strain MHOM/CO84/CL044B as antigen.

Results. The canine population had an average age of 2.5 years; $67.3 \%$ of the dogs were males and the cross-bred animals were the most prevalent constituting $85 \%$ of those samples. On clinical examination the main signs were onicogriphosis $24.3 \%$, lymphadenitis $10 \%$ and skin lesions $5 \%$. The presence of antibodies was observed in $28.1 \%$ of the dogs from sector 8 of Neiva, $28 \%$ in Villavieja, $14.9 \%$ in Rivera, $10 \%$ in Palermo and $5.1 \%$ in Algeciras. An average ratio of five people cohabitating per seropositive dog was observed.

Conclusions. The results reflect the exposure to Leishmania chagasi infection of dogs living in both urban and rural environments in the studied zones, and should encourage health authorities to carry out control measures to prevent the spread of this zoonotic disease.
\end{abstract}

Key words: dog diseases, Leishmania, enzyme-linked immunosorbent assay, Colombia

Los protozoarios intracelulares del género Leishmania afectan al hombre y a otros mamíferos, entre ellos, los cánidos, en los que pueden ocasionar un amplio espectro de procesos patológicos que van desde cuadros cutáneos autorresolutivos hasta procesos viscerales con consecuencias fatales $(1,2)$.

La forma visceral en el hombre es causada por Leishmania chagasi en América y podría asociarse con la presencia simultánea de brotes de leishmaniosis visceral canina en razón a que el perro, debido a su estrecho contacto con el hombre, se constituye en el reservorio doméstico más importante dentro del ciclo de transmisión, compartiendo en zonas endémicas, los nichos ecológicos de reservorios silvestres e insectos vectores (3-8).

La leishmaniosis visceral en los caninos es una parasitosis con repercusión en medicina veterinaria, en la cual la dificultad en el diagnóstico y la baja eficacia de su tratamiento constituyen obstáculos para el control de la enfermedad en zonas endémicas, convirtiéndose en un riesgo para la salud pública, dado el carácter zoonótico del proceso $(4-7,9)$.

\footnotetext{
Correspondencia:

José Fernández M., Universidad de La Salle, Facultad de Medicina Veterinaria, Grupo de Epidemiología y Salud Pública, Calle 68 No. 49-47 Bloque 19, apartamento 503, Bogotá D.C., Colombia.

Teléfono (571) 630 8978; fax: (571) 6772636

josefernandezm@yahoo.com
}

Recibido: 19/07/05; aceptado: 07/03/06
Los focos más importantes de leishmaniosis visceral en Colombia se encuentran distribuidos a lo largo de la cuenca del río Magdalena y sus afluentes en los departamentos de Huila, Tolima, Cundinamarca, Bolívar, Sucre, Córdoba y Santander que corresponden a la distribución de los vectores Lutzomyia longipalpis y Lutzomyia evansi $(8,10)$. En el 2004 se registraron 96 casos humanos de leishmaniosis visceral en el país, de los cuales, cinco correspondieron al departamento del Huila, cuatro de éstos registrados en Neiva (11).

En Colombia, los estudios realizados en perros incluyen los desarrollados en la Costa Caribe, en el corregimiento de Cerro Vidales y en el municipio de Montería, Córdoba, donde se informaron mediante exámenes parasitológicos prevalencias de $8,33 \%$ y $26,17 \%$, respectivamente; de la misma forma, mediante prueba de inmunofluorescencia indirecta se identificaron en los municipios de Ovejas (Sucre), San Andrés de Sotavento (Córdoba) y El Carmen de Bolívar (Bolívar), con prevalencias entre $3,84 \%$ y $9,6 \%$ (12). Vélez et al. en un estudio ecoepidemiológico de leishmaniosis visceral en la comunidad indígena zenú de San Andrés de Sotavento, Córdoba, reportaron el $16 \%$ de los caninos seropositivos mediante la prueba de inmunofluorescencia indirecta (13). Otros trabajos en el interior del país han registrado seroprevalencias de $12,5 \%$ en el municipio de Rovira (Tolima) y de $17,2 \%$ en caninos de 17 veredas del departamento del Huila (12).

Corredor et al. (1989) (10) encontraron seroconversión de caninos centinelas en un rango de 4,4 a 8 meses, después del ingreso a la zona 
de estudio como parte de un programa de vigilancia epidemiológica en el municipio de Ricaurte (Cundinamarca), así mismo se aislaron cepas de campo a partir de los perros seropositivos, que fueron identificadas como $L$. chagasi.

La necesidad de una técnica más eficiente en el diagnóstico de leishmaniosis visceral permitió desarrollar diferentes tipos de pruebas inmunoenzimáticas como la prueba de ELISA con sus diferentes variaciones (14). Para el diagnóstico de la leishmaniosis visceral en perros, esta prueba tiene una sensibilidad de $98 \%$ y una especificidad entre $85 \%$ y $96 \%$ (15). En Colombia se estandarizó una prueba de ELISA con una sensibilidad calculada de $84,5 \%$ y una especificidad de 86,4\% (Vega JC, López MC, Vargas JJ, Ayala MS, Nicholls RS, Bello F et al. Estandarización de la prueba de ELISA para el inmunodiagnóstico de la leishmaniasis visceral canina. En: Agudelo C, editor. I Encuentro de Investigadores en Salud Pública de la Universidad Nacional de Colombia. Bogotá: Instituto de Salud Pública; 2003. p.80-9), técnica que puede ser empleada en estudios seroepidemiológicos necesarios para el diseño de estrategias de prevención y control de leishmaniosis en perros y humanos (15-16).

El propósito de este trabajo fue determinar la prevalencia de anticuerpos IgG contra $L$. chagasi por la prueba de ELISA en sueros de perros, recolectados en los municipios de Rivera, Palermo, Villavieja, Algeciras y en la comuna 8 de Neiva, departamento del Huila, Colombia.

\section{Materiales y métodos \\ Área de estudio}

Se tomaron sueros de perros en los municipios de Rivera, Palermo, Villavieja, Algeciras y en la comuna 8 de Neiva (figura 1). Las condiciones ecológicas corresponden a zonas climáticas de bosque seco tropical con temperatura promedio de $25,8^{\circ} \mathrm{C}$, altitud entre 400 y $1.500 \mathrm{msnm}$ y con casos reportados de leishmaniosis visceral en humanos (12).

\section{Población muestreada}

Se estudiaron 610 perros, muestra aleatoria calculada teniendo en cuenta la población de 1.620 perros, establecida en los registros de vacunación antirrábica del 2003 de la Secretaría Departamental del Huila. Para el cálculo del tamaño de muestra se usó una prevalencia estimada de $17,2 \%$ y un nivel de confianza de $95 \%$. Las muestras fueron tomadas casa a casa.

\section{Encuesta epidemiológica y examen clínico}

Se hizo una encuesta epidemiológica a los propietarios de las mascotas objeto de estudio, con el ánimo de obtener los siguientes datos: nombre del propietario, vereda o barrio, nombre del perro, edad, sexo, raza, historia de vacunación, dieta, condición corporal ( $1=$ delgado, $2=$ bajo de peso, $3=$ =ideal, 4 y $5=$ =sobrepeso) (17) y número de personas que conviven con la mascota. En el examen clínico se evaluó la apariencia general del animal y la presencia de lesiones como úlceras cutáneas, onicogrifosis o hipertrofia de las uñas, áreas depiladas o alopécicas, lesiones eritematosas y linfadenitis.

\section{Recolección de las muestras}

Para la obtención de suero se recolectaron $10 \mathrm{ml}$ de sangre completa por venopunción de la vena cefálica; las muestras se fraccionaron en alícuotas y se almacenaron $\mathrm{a}-20^{\circ} \mathrm{C}$.

\section{Prueba de ELISA}

Se siguieron las condiciones descritas en Vega JC, López MC, Vargas JJ, Ayala MS, Nicholls $\mathrm{RS}$, Bello $\mathrm{F}$ et al. Estandarización de la prueba de ELISA para el inmunodiagnóstico de la leishmaniasis visceral canina. En: Agudelo C, editor. I Encuentro de Investigadores en Salud Pública de la Universidad Nacional de Colombia. Bogotá: Instituto de Salud Pública; 2003. p-80-9.

Como antígeno se emplearon promastigotes de L. chagasicepa MHOM/CO/84/CL044B obtenidos de cultivos en masa que se mantuvieron en medio líquido de Schneider. Se emplearon placas de 96 pozos Dynatech Inmulon I de poliestireno. En cada pozo se agregaron $100 \square$ I de antígeno a una concentración de $7,5 \mu \mathrm{g} / \mathrm{ml}$ en solución reguladora de carbonato-bicarbonato de sodio $0,05 \mathrm{M}, \mathrm{pH}$ 9,6. Se incubaron las microplacas durante tres horas en cámara húmeda a temperatura ambiente, y se lavaron tres veces por cinco minutos con solución reguladora de fosfatos $0,15 \mathrm{M}, \mathrm{pH} 7,4$ con Tween 


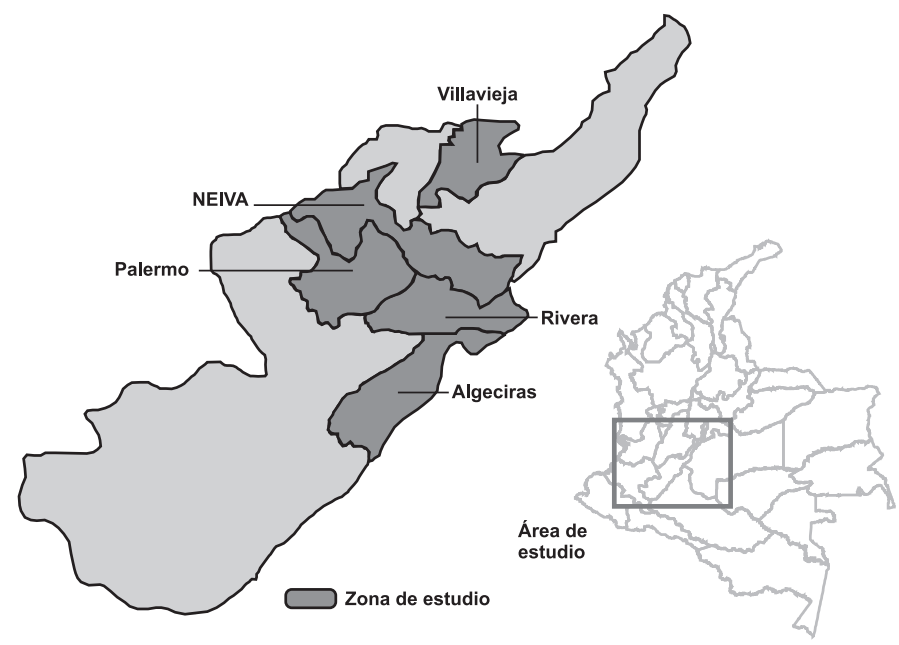

Figura 1. Localización de los municipios muestreados en el departamento del Huila, Colombia, 2004.

20 al 0,05\%. Se dispensaron $100 \mu \mathrm{l}$ de suero de cada perro muestreado, por triplicado en dilución 1:800 en solución reguladora de fosfatos con Tween 20; se incubaron en cámara húmeda a temperatura ambiente durante dos horas y se lavaron tres veces como se describió anteriormente. Se agregaron $100 \mu \mathrm{l}$ de anti-lgG canina marcada con fosfatasa alcalina en dilución 1:6.000 por pozo y se incubaron en cámara húmeda a $4^{\circ} \mathrm{C}$ durante 18 horas. Se lavó nuevamente tres veces y se agregaron a cada pozo 100 니 de 5-para-nitro-fenil-fosfato en proporción 1:1 $(\mathrm{v} / \mathrm{v})$ en solución reguladora de dietanolamina al $10 \%, \mathrm{pH} 9,8$, en una concentración de $1 \mathrm{mg} / \mathrm{ml}$ y las microplacas se incubaron durante 30 minutos a temperatura ambiente. Se agregaron $25 \mu$ de solución de hidróxido de sodio $3 \mathrm{~N}$ para detener la reacción. Las absorbancias se leyeron a una longitud de onda de $405 \mathrm{~nm}$ en un colorímetro UNISKAN I. Se consideraron como sueros positivos aquéllos que mostraron valores de absorbancias iguales a 0,420 .

\section{Análisis de resultados}

Con la información obtenida se construyó una base de datos en el programa Epilnfo 6.04, y se determinaron las frecuencias, que se presentan en los cuadros. Las seroprevalencias se ajustaron mediante la formula $\mathrm{PA}=(\mathrm{p}+\mathrm{E}-1) /(\mathrm{S}+\mathrm{E}-1)(\mathrm{PA}$ : prevalencia ajustada, $p=$ prevalencia observada, $\mathrm{S}=$ sensibilidad, $\mathrm{E}=$ especificidad) metodología descrita por Ahlbom y Norell (18).

\section{Aspectos éticos}

El muestreo se llevó a cabo con el consentimiento previo de los propietarios, que incluyo el derecho a no participar, siguiendo las normas éticas para el manejo de animales establecidas en el Estatuto Nacional de Protección para los Animales, Ley 84 de 1989 y las normas éticas para investigación sin riesgos en comunidades descritas en la Resolución No. 008430 de 1993 del Ministerio de Salud que establece las normas científicas, técnicas y administrativas para la investigación en salud $(19,20)$. La investigación fue aprobada por el Comité de Investigación con Animales del Instituto Nacional de Salud.

\section{Resultados}

En la población canina muestreada, $67,2 \%$ eran machos y $32,8 \%$ hembras; $59,2 \%$ de los perros tenían menos de dos años, con una edad promedio de 2,5 años; la raza más frecuente correspondió a la mestiza con $85,2 \%$. Con respecto al manejo sanitario y nutricional de los animales, $44 \%$ no registró historia alguna de vacunación, sólo el $34,9 \%$ había recibido tratamiento antiparasitario recientemente y $84,9 \%$ consumía únicamente dietas caseras. 
Cuadro 1. Descripción de la población canina seropositiva evaluada mediante prueba de ELISA en cinco municipios de Huila, Colombia, 2004.

\begin{tabular}{|c|c|c|}
\hline Variable & $\mathbf{n}$ & $\%$ \\
\hline Población canina & 1.620 & \\
\hline Total de perros muestreados & 610 & \\
\hline Positivos a ELISA & 118 & 19,34 \\
\hline Seroprevalencia ajustada & & 8,1 \\
\hline IC 95\% & & $5,9-10,2$ \\
\hline \multicolumn{3}{|l|}{ Raza } \\
\hline Mestiza & 95 & 80,5 \\
\hline French Poodle & 5 & 4,2 \\
\hline Boxer & 3 & 2,5 \\
\hline Pincher & 2 & 1,7 \\
\hline Otras & 13 & 10 \\
\hline \multicolumn{3}{|l|}{ Sexo } \\
\hline Machos & 81 & 68,6 \\
\hline Hembra & 37 & 31,4 \\
\hline Relación macho/hembra & $2: 1$ & \\
\hline \multicolumn{3}{|l|}{ Edad } \\
\hline Promedio (años) & $3,1( \pm 2,5)$ & \\
\hline Edad más frecuente & 2 & \\
\hline \multicolumn{3}{|l|}{ Sintomatología } \\
\hline Asintomáticos & 88 & 75 \\
\hline Sintomáticos & 30 & 25 \\
\hline \multicolumn{3}{|l|}{ Vacunación } \\
\hline Ninguna vacuna & 39 & 33 \\
\hline Antirrábica & 76 & 65 \\
\hline No responde & 3 & 2 \\
\hline \multicolumn{3}{|l|}{ Tratamiento antiparásito } \\
\hline Sin tratamiento & 65 & 55,6 \\
\hline Con tratamiento & 52 & 44,4 \\
\hline \multicolumn{3}{|l|}{ Alimentación } \\
\hline Casera & 94 & 80 \\
\hline Mixta & 13 & 11 \\
\hline Concentrado & 10 & 8,5 \\
\hline \multicolumn{3}{|l|}{ Procedencia } \\
\hline Del mismo lugar & 84 & 71 \\
\hline Otro & 34 & 29 \\
\hline \multicolumn{3}{|l|}{ Personas convivientes } \\
\hline Total & 493 & 100 \\
\hline Adultos & 277 & 56 \\
\hline 10 a 18 años & 95 & 19 \\
\hline Niños & 124 & 25 \\
\hline
\end{tabular}

La condición corporal de las mascotas fue ideal (condición 3 ) en 50,8\%; bajo de peso (condición 2), 46,2\%, y delgado (condición 1), 3\%; no se observaron animales con sobrepeso (condiciones 4 y 5). Teniendo en cuenta la sintomatología compatible con leishmaniosis visceral canina, $24,3 \%$ de la población muestreada presentaba onicogrifosis, $10 \%$ linfadenitis, $5 \%$ lesiones cutáneas y 2,7\% disminución del apetito.

Se encontraron 2.619 personas conviviendo con los perros muestreados, $77,5 \%$ mayores de 10 años y $22,5 \%$ menores de 10 años, lo cual representa una proporción general de cuatro personas convivientes por perro y, en el caso de la población infantil, de un niño conviviente por perro.

Se observó la presencia de IgG contra L. chagasi en 118 de los 610 sueros evaluados (cuadro 1). Las seroprevalencias ajustadas por municipio muestreado se observan en el cuadro 2; la totalidad de las muestras positivas recolectadas en Neiva y Rivera provenían de la zona urbana, mientras que en Algeciras la totalidad de sueros positivos provenían de zonas rurales. En el municipio de

Cuadro 2. Seroprevalencia de leishmaniosis visceral canina obtenida en municipios de Huila, Colombia, 2004.

\begin{tabular}{llcc}
\hline Municipio & \multicolumn{1}{c}{ Variable } & $\mathbf{n}$ & $\%$ \\
\hline Neiva & & & \\
& Total muestras & 153 & \\
& Perros positivos & 43 & 28,1 \\
& Seroprevalencia ajustada & & 20,45 \\
& IC 95\% & & $14,05-26,08$ \\
Villavieja & & 152 & \\
& Total muestras & 41 & 28 \\
& Perros positivos & & 20,3 \\
& Seroprevalencia ajustada & & $13,9-26,7$ \\
& IC 95\% & & \\
Rivera & Total muestras & 138 & 14,9 \\
& Perros positivos & 20 & 1,8 \\
& Seroprevalencia ajustada & & $0,4-4,0$ \\
& IC 95\% & & \\
Palermo & Total muestras & 90 & \\
& Perros positivos & 9 & 10 \\
Algeciras & Total muestras & 78 & \\
& Perros positivos & 4 & 5,1 \\
\hline
\end{tabular}

Cuadro 3. Discriminación por grupos de edad de la población humana que convive con perros seropositivos a leishmaniosis visceral en los municipios muestreados de Huila, Colombia, 2004.

\begin{tabular}{lccc}
\hline Municipio & \multicolumn{3}{c}{$\begin{array}{c}\text { Promedio de personas convivientes } \\
\text { con un perro seropositivo }\end{array}$} \\
\cline { 2 - 4 } & $<\mathbf{1 0}$ años & $\mathbf{1 0}$ a $\mathbf{1 8}$ años & $>\mathbf{1 8}$ años \\
\hline Neiva & 1,5 & 1,2 & 3,1 \\
Villavieja & 0,8 & 0,7 & 19 \\
Rivera & 0,7 & 0,4 & 1,6 \\
Palermo & 0,6 & 0,4 & 2 \\
Algeciras & 2,25 & 1 & 4 \\
Total & $\mathbf{1 , 1}$ & $\mathbf{0 , 8}$ & $\mathbf{2 , 3}$ \\
\hline
\end{tabular}


Villavieja, 21,9\% de las muestras positivas provenían de zona rural y $78,1 \%$ del casco urbano; en Palermo, $60 \%$ provenía de zona urbana y $40 \%$ de zona rural (cuadro 2).

Como se describe en el cuadro 1, en la población con anticuerpos contra $L$. chagasi, el promedio de edad fue de 3,1 años y $80,5 \%$ correspondió a perros mestizos. En el examen clínico, $75 \%$ de los animales seropositivos fueron asintomáticos y las manifestaciones clínicas más frecuentes en la población sintomática fueron: onicogrifosis, 24,8\%; linfadenitis, 12,8\%; alopecia, 5,2\%, y emesis, $4,3 \%$. Con respecto a la procedencia de los perros seropositivos, debe tenerse en cuenta que $29 \%$ fueron introducidos al área de estudio.

Los resultados obtenidos muestran que 493 personas se encuentran en contacto con los perros seropositivos (cuadro 1). En la población mayor de 18 años del municipio de Algeciras, cuatro personas en promedio convivían con un perro seropositivo; similar situación se presentó en Neiva donde tres personas adultas en promedio convivían con un perro seropositivo (cuadro 3). Los municipios de Palermo, Villavieja y Rivera mostraron promedios similares de contacto de la población adulta con perros con anticuerpos (cuadro 3).

En la población humana menor de 10 años, la distribución fue similar a la observada en la población adulta en cada municipio pero disminuyó el promedio de conviventes por perro seropositivo (cuadro 3). Por otro lado, el promedio de personas entre 10 y 18 años de edad que convivían con un perro seropositivo fue menor con respecto al promedio de convivientes en los demás grupos de edad.

\section{Discusión}

Las áreas objeto de estudio se encuentran ubicadas por debajo de los $1.750 \mathrm{msnm}$ y tienen las condiciones geoecológicas para la transmisión y la proliferación de leishmaniosis visceral, hecho que se correlaciona con lo reportado por Vélez et al. (13) y Corredor et al. (21) quienes incluyeron al departamento del Huila como uno de los focos en Colombia de leishmaniosis visceral $(10,13)$.

En este estudio se observó la presencia de perros infectados tanto en zonas rurales como urbanas del departamento del Huila, lo cual se podría explicar por el desplazamiento de la población y sus mascotas a las ciudades, a la presencia de reservorios y vectores a nivel urbano y a la circulación de los perros entre los ambientes silvestres y domésticos, como lo describen Paranhos-Silva et al. (22) y Silva et al. (23).

Las diferencias de seroprevalencia ajustadas obtenidas en Neiva $(20,4 \%)$ y Villavieja $(20,3 \%)$ con respecto a los demás municipios, podrían indicar variaciones en la densidad tanto de vectores como de perros, similar a lo descrito por Busch et al. (24) y Ciaramella et al. (25); sin embargo, los porcentajes de positividad de anticuerpos obtenidos en los municipios de Algeciras, Palermo y Rivera son similares a las obtenidos en otros estudios realizados en zonas rurales de Colombia (12).

Teniendo en cuenta las variables asociadas con la leishmaniosis visceral en perros de zonas endémicas, el sexo no se constituyó en un determinante negativo debido a que la proporción de hembras y machos seropositivos fue similar; no obstante, el número de machos en la población es mayor al de hembras, probablemente por la preferencia de los propietarios a la tenencia de machos. La edad es un indicador del grado de transmisión de la infección; Moreno y Alvar (26) reportaron que en los casos de leishmaniosis visceral canina las prevalencias son mayores en animales de hasta tres años de edad.

Los resultados obtenidos muestran un promedio de edad para la población general de 2,5 años y para la población seropositiva de 3,1 años, datos que permiten inferir que las prevalencias obtenidas se relacionan con una población joven. En Colombia se ha descrito que la supervivencia de perros infectados en focos endémicos no es mayor a dos años de edad (21). Teniendo en cuenta que el promedio de edad en los animales seropositivos fue mayor, podría pensarse que estos animales se han infectado de forma tardía o se mantienen en el estado de asintomáticos que correspondió al $75 \%$ de la población seropositiva muestreada.

Aunque la linfadenitis y la onicogrifosis son signos inespecíficos que se pueden presentar en 
diferentes patologías caninas, cuando estos signos se presentan con el antecedente epidemiológico de vivir o haber vivido en zona endémica, podría sugerir la presencia de leishmaniosis visceral canina, como lo describió Ciaramela et al. (25).

En Colombia, según Vélez et al. (13), la mortalidad de perros en áreas endémicas puede alcanzar $25 \%$, cifra similar a la obtenida en los perros seropositivos sintomáticos muestreados, los cuales tendrían una mayor probabilidad de morir a causa de la infección. La talla, raza y peso de los animales no constituyeron variables importantes, como fue descrito por Moreno y Alvar (26).

Los casos de leishmaniosis visceral canina en áreas endémicas donde de forma concomitante se presenta la entidad en humanos han sido reportados desde los primeros aislamientos realizados en 1908 por Nicolle y Compte en Túnez (24).

En Colombia, en el 2003 se registraron 121 casos en humanos que disminuyeron a 96 en el 2004; sin embargo, en la zona de estudio el número de casos pasó de dos en el 2003 a cinco en el 2004 (11). Es posible que exista una asociación entre leishmaniosis visceral humana y canina, ya que las mascotas se convierten en reservorios de la entidad en los ambientes domésticos en donde normalmente habitan generando un factor de riesgo para las personas que conviven con ellas siempre y cuando exista la presencia del vector L. longipalpis, el cual ha sido descrito en focos bien estudiados que incluyen la zona de estudio $(11,21,22,27,28)$.

Aunque se considera que los perros de las áreas rurales son los más afectados, la infección se ha reportado en regiones urbanas y suburbanas de países como Brasil en las ciudades de Boa Vista, Santarém, Terezina, São Luis, Natal, Aracajú, Montes Claros, Belo horizonte, Araçuaí, Sabará, Perdões, Rio de Janeiro y Cuiabá así como en Venezuela en el área úrbana de Valencia en el estado de Carabobo y en la isla de Margarita (14, 29-31).

En Colombia son pocos los trabajos realizados con respecto a la situación de la leishmaniosis visceral canina en zonas urbanas; sin embargo, en este estudio se encontró una seroprevalencia ajustada de $20,45 \%$ en Neiva, dato que contrasta con los resultados obtenidos a nivel rural donde se han observado seroprevalencias que varían entre $3,8 \%$ y $17 \%$ en diferentes zonas de Colombia (12). La sensibilidad del $84,5 \%$ y la sensibilidad del $86,4 \%$ de la prueba de ELISA no permitió ajustar las seroprevalencias de los municipios de Algeciras y Palermo (cuadro 2).

Actualmente, se considera que el patrón de transmisión de las leishmaniosis está cambiando en Colombia $(28,32)$, hecho que se evidencia con la aparición de brotes de leishmaniosis cutánea en personas que habitaban ciudades como Sincelejo (28), Bucaramanga (33), Remedios (34), Villeta y Quebradanegra (35) y de igual forma con la demostración de la presencia de vectores incriminados en la transmisión en zonas periurbanas de ciudades como Medellín (32).

En la actualidad, se discute cómo la leishmaniosis visceral se encuentra en un nítido proceso de transición epidemiológica, identificado por una incidencia creciente en áreas endémicas y su expansión geográfica a zonas no endémicas, incluso a zonas urbanas (14). En Colombia, ya se reportan casos de leishmaniosis visceral en niños sin nexos epidemiológicos con zonas endémicas rurales en Sincelejo (28) y de la misma forma, en Neiva, donde se desarrolló parte de este estudio, se reportaron dos casos en el 2003 y cuatro casos en el 2004, en la población infantil (11).

Todos estos factores hacen sospechar que los perros pueden tener un papel preponderante dentro de la nueva ecoepidemiología de la leishmaniosis visceral en zonas urbanas y, específicamente, en el departamento del Huila, más aún, si se tiene en cuenta que se observaron parásitos del género Leishmania en cultivos preparados a partir de los tejidos de dos perros seropositivos procedentes de la comuna 8 de Neiva y que el perro es considerado el reservorio doméstico más importante, con capacidad de establecer patrones enzoóticos silenciosos debido a su mayor probabilidad de contacto con los vectores y reservorios silvestres en su hábitat natural (24). 
Sin embargo, hay que tener en cuenta que la importancia del perro como reservorio no debe asumirse solamente en zonas endémicas; en zonas no endémicas como los Estados Unidos, se reconoce hoy la importancia y el impacto a nivel de salud pública de esta enfermedad emergente en los perros (27).

En este estudio, el mayor promedio de personas en contacto por perro seropositivo se observó en el municipio de Algeciras. Sin embargo, la seropositividad fue la menor del estudio, posiblemente a causa de que el área de muestreo se encontraba a nivel periurbano y la densidad de la población por domicilio era alta.

En Neiva la seroprevalencia y el promedio de convivientes por perro seropositivo fueron altos; si se tiene en cuenta la alta densidad de población tanto humana como canina por unidad de área a nivel urbano, el riesgo potencial de transmisión cuando existe la presencia de vectores adecuados podría incrementarse (32). Lo anterior podría explicar el aumento de la incidencia de casos humanos observados en Neiva.

Es necesario tener en cuenta que las poblaciones en riesgo de contraer la infección en áreas donde confluyen tanto reservorios como vectores, son principalmente niños y adultos inmunocomprometidos. En este estudio, aunque no se contempló el estado inmunológico de las personas, se observa que el promedio de personas adultas convivientes mayor de 18 años es el más alto para todas las áreas muestreadas y esta seguido por la población infantil menor de 10 años, grupo de edad en la cual se reportan la mayoría de casos de leishmaniosis visceral $(15,16,28)$.

De esta forma, el presente trabajo confirma la endemicidad de la infección en los caninos de los municipios muestreados y alerta sobre la presencia de perros seropositivos en zonas urbanas. Esta situación sugiere el urgente fortalecimiento de los programas de prevención y control de leishmaniosis visceral en el Sistema General de Seguridad Social en Salud, en especial, en Neiva y Villavieja donde un alto porcentaje de perros que habitan las áreas urbanas muestran la presencia de anticuerpos frente a la infección por L. chagasi.
En Latinoamérica la urbanización de las leishmaniosis es un fenómeno creciente y el riesgo de contraer la infección puede incrementarse si no se controla el contacto de los vectores y la población humana con reservorios infectados como los perros.

\section{Agradecimientos}

A los estudiantes de Medicina Veterinaria de la Universidad de La Salle; a Martha Páez, Arnulfo Abril y John Penagos, por su colaboración en la recolección de material a nivel de campo. A los funcionarios de salud ubicados en el Centro de Zoonosis de Neiva y en los Centros de Salud de los municipios del área de estudio, por su colaboración en sensibilización de la comunidad y en la recolección del material de campo. A la comunidad de los municipios estudiados por facilitar las mascotas que permitieron llevar a cabo este estudio.

\section{Conflicto de intereses}

En la realización del presente trabajo no se presentó ningún conflicto de interés financiero, político ni académico.

\section{Financiación}

El presente estudio se realizó con el apoyo económico de la Universidad de La Salle, la Universidad Nacional de Colombia, el Instituto Nacional de Salud y la Secretaría Departamental de Salud del Huila.

\section{Referencias}

1. Ordóñez C, Lerner E. Maxadilán: un potente vasodilatador cutáneo aislado de la especie Lutzomia longipalpis, vector de la leishmaniasis visceral. Biomédica 1996;16:58-61.

2. Palacios R, Valderrama L. Leishmaniosis: biología e inmunología. Innovación y Ciencia 1998;7:18-23.

3. Acedo-Sánchez C, Morillas-Márquez F, SánchizMarín MC, Martín-Sánchez J. Changes in antibody titers against Leishmania infantum in naturally infected dogs in Southern Spain. Vet Parasitol 1998;75:1-8.

4. Dye C. The logic of visceral leishmaniosis control. Am J Trop Med Hyg 1996;55:125-30.

5. Pearson RD, Cox G, Jeronimo SM, Castracane J, Drew JS, Evans T et al. Visceral leishmaniasis: a model for infection-induced cachexia. Am J Trop Med Hyg 1992;47:8-15. 
6. Sherlock IA. Ecological interactions of visceral leishmaniasis in the state of Bahia, Brazil. Mem Inst Owaldo Cruz 1996:91:671-83.

7. Tesh RB. Control of zoonotic visceral leishmaniasis: is it time to change strategies? Am J Trop Med Hyg 1995;52:287-92.

8. Travi BL, Osorio Y, Becerra MT, Adler H. Dynamics of Leishmania chagasi infection in small mammals of the undisturbed and degraded tropical dry forests of northern Colombia. Trans R Soc Trop Med Hyg 1998;92:275-8.

9. Zaffaroni E, Rubaudo L, Lanfranchi P, Mignone W. Epidemiological patterns of canine leishmaniasis in Western Liguria (Italy). Vet Parasitol 1999;81:11-9.

10. Corredor A, Gallego JF, Tesh RB, Morales A, de Carrasquilla CF, Young DG et al. Epidemiology of visceral leihsmaniasis in Colombia. Am J Trop Med Hyg 1989;40:480-6.

11. Subdirección de Vigilancia y Control en Salud Pública. Informe de Vigilancia y control, Colombia, 2004. Bogotá: Instituto Nacional de Salud, 2005. p.7-8.

12. Fernández J, Charry T, Bello F, Escovar J, Lozano C, Ayala $\mathbf{M}$ et al. Prevalencia de leishmaniasis visceral canina en municipios de Huila-Colombia. Rev Salud Pública 2002;4:278-85.

13. Vélez ID, Travi BL, Gallego J, Palma GI, Agudelo SP, Montoya $\mathbf{J}$ et al. Evaluación ecoepidemiológica de la leishmaniasis visceral en la comunidad indígena Zenú de San Andrés de Sotavento, Córdoba: primer paso para su control. Revista Colombiana de Entomología 1995;21:111-22.

14. Alexandre W, Dias $\mathbf{P}$. Reflexões sobre a qualidade do diagnóstico da leishmaniose visceral canina em inquéritos epidemiológicos: o caso da epidemia de Belo Horizonte, Minas Gerais, Brasil, 1993-1997. Cad Saúde Pública 2004;20:259-65.

15. Travi BL, Tabares CJ, Cadena H, Ferro CR, Osorio J. Canine visceral leishmaniasis in Colombia: relationship between clinical and parasitological status and infectivity for sand flies. Am J Trop Med Hyg 2001;64:119-24.

16. Moreira ED Jr, de Souza VM, Sreenivasan M, Lopes NL, Barreto RB, De Carvalho L. Peridomestic risk for canine leishmaniasis in urban dwellings: New findings from a prospective study in Brasil. Am J Trop Med Hyg 2003;69:393-7.

17. Case LP, Carey DP, Hirakawa DA. Desarrollo y tratamiento de la obesidad. En: Álvarez J, editor. Nutrición canina y felina. Manual para profesionales. Madrid: Harcourt Brace; 1997. p.247-68.

18. Ahlbom A, Norell S. Introduction to modern epidemiolgy. Segunda edición. Chestnut Hilma: Epidemiology Resources Inc.; 1984. p.25.
19. Congreso de la República de Colombia. Ley 084 de 1989. Estatuto Nacional de Protección de los animales. Diario Oficial, Año CXXVI. N.39120.27; 1989. p.1-14.

20. Ministerio de Salud. República de Colombia. Resolución No. 008430 de 1993. Investigación en Salud; 1993. p.1-20.

21. Corredor A, Kreutzer RD, Tesh RB, Boshell J, Palau $\mathbf{M}$, Cáceres $\mathbf{E}$ et al. Distribution and etiology of leishmaniasis in Colombia. Am J Trop Med Hyg 1990;42:20614

22. Paranhos-Silva M, Nascimento EG, Melro MC, Oliveira GG, dos Santos WL, Pontes-de-Carvalho LC et al. Cohort study on canine emigration and Leishmania infection in an endemic area for American visceral leishmaniasis. Implications for the disease control. Acta Trop 1998;69:75-83.

23. Silva ES, Gontijo CM, Primez C, Fernandez O, Brasil RP. Short report: detection of Leishmania DNA by polymerase chain reaction on blood samples from dogs with visceral leishmaniasis. Am J Trop Med Hyg 2001;65:896-8.

24. Busch I, Camargo M, Villanova A, Reichmann A, Andrade E, Tolezang J. Inquerito serológico para pesquisa de leishmaniose visceral em populacio canina urbana do municipio de Sao Paulo Brasil. Rev Inst Med Trop Sao Paulo 1983;25:310-7.

25. Ciaramella P, Oliva G, de Luna RD, Gradoni R, Ambrosio R, Cortese $L$ et al. A retrospective clinical study of canine leishmaniasis in 150 dogs naturally infected by Leishmania infantum. Vet Rec 1997; 141:539-43.

26. Moreno J, Alvar J. Canine leishmaniasis epidemiological risk and the experimental model. Trends Parasitol 2002;18:399-405.

27. Rosypal AC, Zajac AM, Lindsay DS. Canine visceral leishmaniasis and its emergence in the United States. Vet Clin North Am Small Anim Pract 2003;33:921-37.

28. Bejarano EE, Uribe S, Rojas W, Vélez ID. Phlebotomine sand flies (Diptera: Psychodidae) associated with the appearance of urban leishmaniasis in the city of Sincelejo, Colombia. Mem Inst Oswaldo Cruz 2002; 97:645-7.

29. Aguilar C, Fernández E, Fernández R, Cannova D, Ferrer E, Cabrera Z et al. Visceral leishmaniasis in Venezuela. Mem Inst Oswaldo Cruz 1998;93:15-6.

30. Cabrera MA, Paula AA, Camacho LA, Marzochi C, Navier SC, Silva AV et al. Canine visceral leishmaniasis in Barra de Guaratiba, Rio de Janeiro, Brasil: Assessment of risk factors. Rev Inst Med Trop Sao Paulo 2003;45:79-83.

31. Zerpa O, Ulrich M, Benitez M, Avila C, Rodriguez V, Centeno $\mathrm{M}$ et al. Epidemiological and immunological aspects of human visceral leishmaniasis on Margarita 
Island, Venezuela. Mem Inst Oswaldo Cruz 2002;97:1079-83.

32. Agudelo LA, Uribe J, Sierra D, Ruiz F, Vélez ID. Presence of American cutaneous leishmaniasis vectors surrounding the city of Medellin, Colombia. Mem Inst Oswaldo Cruz 2002;97:641-2.

33. Sandoval CM, Angulo VM, Gutierrez R, Muñoz G, Ferro C. Especies de Lutzomyia (Dipeta:Psychodidae) posibles vectores de leishmaniasis en la ciudad de Bucaramanga, Santander, Colombia. Biomédica 1998;18:161-8.
34. Vargas GS, Alvarez G, Wolf M, López Y, Gómez ME. Estudio de un foco de leishmaniosis en dos barrios de Remedios, Antioquia, 1990. Boletín Epidemiológico de Antioquia 1991;16:48-59.

35. Pardo RH, Farieta S, Munstermann LE, Ferro C. Estudio preliminar de los flebótomos de Villeta y Quebradanegra, Cundinamarca: sus implicaciones en salud pública. Biomédica 1996;16:293-302. 